\title{
NUEVOS DESARROLLOS EN LA APLICACIÓN DE MATERIALES COMPÓSITOS EN CONSTRUCCIONES: SISTEMAS DE RESINAS A BASE ACUOSA "BET ON TEX IPN" Y SU APLICACIÓN EN TECNOLOGÍAS FRP Y FRCM
}

\section{NEW DEVELOPMENT IN COMPOSITE APPLICATIONS FOR CONSTRUCTIONS: BETONTEX IPN WATER RESINS SYSTEMS FOR FRP AND FRCM TECHNOLOGIES}

\author{
LINO CREDALI', GIANLUCA USSIA ${ }^{2}$ \\ 1 Ardea Progetti e Sistemi srl. Italia. ardea.credali@betontex.it \\ 2 Ardea Progetti e Sistemi srl. Italia. Gianluca.Ussia@betontex.it
}

Los materiales compósitos formados principalmente por fibras de carbón y matrices de resinas poliméricas epoxi han sido utilizados por más de 30 años, alcanzando un alto nivel técnico en todo el mundo. A pesar de los extraordinarios resultados obtenidos con este tipo de resinas, algunas de sus características han sido fuertemente criticadas, teniendo como consecuencia algunas limitaciones en su aplicación y en la definición de soluciones de diseño. Las restricciones de las aplicaciones de las tecnologías basadas en resinas epoxi se deben principalmente a su ausencia de permeabilidad a la humedad, su alta sensibilidad a la humedad durante la aplicación, sus escasas propiedades térmicas con un valor de T.g. de $100^{\circ} \mathrm{C}$ y su falta de resistencia a la quema.

En nuestra empresa Ardea, hemos llevado a cabo una larga investigación sobre este tema y hemos introducido en el mercado de la construcción un nuevo sistema de resinas a base acuosa, Betontex IPN, los cuales están basados en estructuras poliméricas interpenetradas. Betontex IPN es un sistema de resinas de dos componenes: el Componente $A$ es una sustancia polimérica emulsionada en agua y el Componente B es una segunda fase polimérica presente en un relleno inorgánico microcristalino. Después del mezclado $\mathrm{e}$ homogenizado, el material resultante es permeable a la humedad y resistente a la quema (de acuerdo a la normativa UNI 9177), con una resistencia térmica más allá de los $180^{\circ} \mathrm{C}$ y una temperatura de degradación mayor a $600^{\circ} \mathrm{C}$. Las propiedades de éstas resinas en combinación con refuerzos de fibras de carbón, aramida y vidrio han sido evaluadas en estructuras de mampostería y concreto. Las resinas IPN muestran una gran gama de propiedades interesantes y pueden ser usadas como tecnología FRP en lugar de las resinas epoxi, o como tecnología FRCM, en donde el IPN actúa como promotor de adhesión entre las fibras y el mortero. Otras propiedades de las resinas IPN incluyen buenas propiedades mecánicas, buena adhesión a las fibras, alta compatibilidad y buena adhesión a morteros de cal, concreto, mampostería, madera y mármol. El sistema de resinas IPN puede también ser usado en condiciones húmedas y aplicado sobre superficies mojadas. En los últimos años, las nuevas tecnologías IPN han sido ampliamente usadas para la rehabilitación de estructuras dañadas como consecuencia de los terremotos de 2009 (L'Aquila, Italia) y 2012 (Emilia Romagna, Italia).

PALABRAS CLAVE: matrices, ipn, compósitos ipn, resinas de agua frp, frcm en construcción ABSTRACT
Composite materials mainly based on carbon fibers and epoxy resins matrices are used in constructions since 30 years and have reached in a very short time a high technical level in all the world. Besides this extraordinary result, some lack of properties of epoxy resins have being criticized, bringing some limitations in applications and in the definition of designing solutions. Restrictions in applications of epoxy-based technologies are due to absence of resins' moisture permeability, high sensitivity to humidity during application, low thermal properties with a $\mathrm{Tg}$. value of $100{ }^{\circ} \mathrm{C}$ and a lack of fire resistance. As Ardea Company, we have developed a long research work on this topic and a new water resin system, Betontex IPN, based on interpenetrated polymer network structures, has been introduced on the construction market. Betontex IPN are a two components resin system: Component $A$, a polymeric substance in water emulsion and component $B$, a second polymer phase supported on active inorganic micro crystals filler. After mixing and hardening, the resulting material is permeable to water moisture and resistant to fire (according to UNI 9177), with a thermal resistance over $180^{\circ}$ $C$ and ultimate degradation temperature over $600^{\circ} \mathrm{C}$.

The properties of the resins in combination with carbon, aramide and glass fiber reinforcements have been tested in masonry and concrete structures. IPN resins show a large range of many interesting properties and may be used as FRP technology, in place of epoxy resins, or as FRCM technology, where IPN acts as adhesion promoter between fibers and mortar. Other properties of IPN resins are: high mechanical properties; good adhesion to fibers, high compatibility and good adhesion to lime mortar, concrete, masonry, wood and marble. The IPN resin system may be used also in humid conditions and applied over wet surface. In the last years the new IPN technologies have been largely applied for rehabilitation of damaged structures after earthquakes of 2009 (L'Aquila) and 2012 (Emilia Romagna-Italy).

KEYWORDS: matrices, ipn, composites ipn, water resin frp, frcm in construction 
INTRODUCCIÓN

Los materiales compósitos (FRP, por sus siglas en inglés) - usados en la construcción de edificios principalmente como rehabilitación estructural - están formados por fibras de carbon y/o aramida y/o vidrio en combinación con matrices poliméricos, generalmente epoxi, modificadas especifícamente para estas aplicaciones.

Los beneficios principales de estas tecnologías incluyen una alta resistencia a la tracción, baja gravedad específica e insensibilidad a la corrosión. Además, no provocan aumentos significativos en el peso, no son invasivos, pueden ser aplicados en superficies curvas debido a su capacidad de adaptarse a nuevas formas y se pueden ocultar fácilmente de la vista mediante recubrimientos externos de enlucido. Su uso permite llevar a cabo la consolidación rápida y fácilmente, aún sin interrumpir las actividades relacionadas al uso de la estructura que debe ser reforzada.

Estas tecnologías han sidoaplicadas y son aún las preferidas en situaciones de gran dificultad como lo son casos de reparación, rehabilitación, mejoramiento o cambio del uso del edificio o en caso de edificios dañados por terremotos. En Italia, ésta es definitivamente la tecnología más importante usada en las zonas sísmicas como el Aquila (terremoto 2009) y Emilia Romagna (terremoto 2012). Los materiales FRP han sido introducdos y han sido también ampliamente usados para el refuerzo de edificios de patrimonio histórico de alto valor artístico (basílicas, iglesias, castillos), que requieren tecnologías más sofisticadas, materiales de alta calidad y alta calidad de diseño.

A pesar del excelente rendimiento mecánico demostrado en 20 años de aplicación, existen muchas críticas relacionadas con el uso de matrices orgánicas de naturaleza epoxi. De hecho, el uso de resinas epoxi ha sido criticado con frecuencia en relación a la consolidación de construcciones de grande interés histórico y artístico debido a la baja compatibilidad de esta resina con la estructura y la falta de porosidad.

El bajo valor de velocidad de transmisión de vapor de agua (MVTR, por su nombre en inglés Moisture Vapor Transmission Rate) de las resinas epoxi puede ser una limitación para su aplicación debido a la posible formación de condensación o retención de agua, la cual puede provocar un gran daño a las estructuras, decoraciones y frescos si éstos están presentes. Además, el uso de resinas epoxi requiere una especial atención en edificios en los cuales es necesario asegurar un adecuado rendimiento estructural bajo fuego.

Otro punto crítico de este tipo de resinas es su baja resistencia térmica debido a sus bajos valores de $\mathrm{Tg}$ (temperatura de transición vítrea de la matriz), los cuales tienen como consecuencia una fuerte disminución del rendimiento mecánico al superar esta temperatura y cuando la resina epoxi es aplicada mediante "laminación manual" o "laminación in situ", que es la técnica estándar de aplicación en la construcción. Usualmente para las resinas epoxi laminadas in situ, el valor de Tg está entre $90^{\circ} \mathrm{C}$ y $100^{\circ} \mathrm{C}$, una laminación que requiere un refuerzo FRP para ser protegido por materiales adecuados, similar a lo que pasa con estructuras de acero.

Para superar estas limitaciones, nuevas tecnologías empleando matrices inorgánicas en lugar de las resinas epoxi, como morteros de cemento y morteros de cal, están actualmente siendo investigadas y muy cerca de ser consideradas en aplicaciones reales. Estas tecnologías, que son frecuentemente identificadas como FRCM (por su nombre en inglés Fibre Reinforced Cementitious Matrix), son el objeto de varios estudios en muchas universidades italianas y estadounidenses. La tecnología FRCM parace ser muy apropiada para mampostería y estructuras de paredes para las cuales se requiere una alta respirabilidad y ductibilidad.

Por nuestra parte, Ardea ha considerado este problema por muchos años mediante un extenso programa de investigación y mediante el desarrollo de una formulación completamente nueva de resinas, las Resinas Betontex IPN, las cuales pueden ser consideradas una solución a muchos de los problemas anteriormente mencionados.

Este producto completamente nuevo está constituido por matrices poliméricas de nueva formulación que están formadas por resinas a base acuosa interpenetradas en una estructura con rellenos minerales activos, definido como IPN (por su nombre en inglés, Interpenetrated Polymer Network).

Estas nuevas resinas poseen una alta porosidad y buena MVRT, son productos a prueba de fuego (no se queman) y presentan alta resistencia térmica, todas estas propiedades de gran interés no sólo para mampostería sino también para estructuras RC.

Una de las principales características de esta nueva resina IPN es la posibilidad de ser usada para ambas tecnologías FRP y FRCM, de tal manera que nuestras pruebas toman en cuenta la investigación para aplicaciones en estructuras de 
concreto y en construcciones de mampostería. Como esta nueva resina ha sido aplicada en trabajos reales desde al menos dos años, algunos ejemplos de aplicaciones son reportados en este artículo.

Una gran parte de nuestra investigación, las pruebas de laboratorio de las propiedades mecánicas y las aplicaciones estructurales se realizaron en la Universidad de Bolonia por el grupo de investigación del Profesor Marco Savoia en el Centro de Investigación de Edificios y Construcción (CIRI). Además, pruebas mecánicas precedentes fueron realizadas en los laboratorios DICAM de la Universidad de Bolonia (1) y la Universidad de Florencia (2).

Además, una contribución importante de este trabajo fue desarrollada por el grupo de Diseño de Materiales del Departamento de Ingeniería "Enzo Ferrari” de la Universidad de Módena (caracterizaciones fisicoquímicas y morfológicas de los materiales).

\section{CRITERIOS DE LOS MATERIALES COMPÓSITOS}

Se debe tener en mente una primera consideración: es bien sabido por cualquier técnico que trabaje en el área de compósitos, que las propiedades mecánicas del FRP dependen de una simple relación, referida al refuerzo unidireccional ideal como reportado en figura 1.

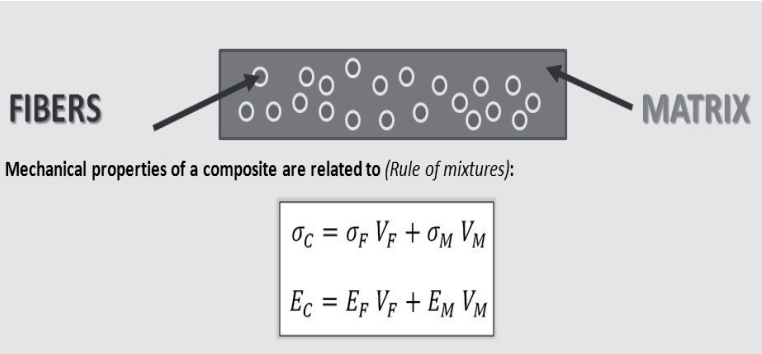

Figura 1. Relación de propiedades mecánicas para un refuerzo compósito unidireccional ideal.

Esta simple relación, normalmente usada para definir las propiedades de compósitos en construcción y también reportada en los documentos CNR DT200/2004 (3) y Ceb/Fib 14/2001 (4), puede ser aplicada sólo si la siguiente condición de congruencia es respetada:

$$
\varepsilon_{c}=\varepsilon_{f}=\varepsilon_{m}
$$

Donde $\varepsilon$ es respectivamente la deformación del: $\varepsilon_{c}$ compósito, $\varepsilon_{f}$ fibra y $\varepsilon_{m}$ matriz, lo que significa una perfecta adhesión entre los dos componentes.
En la práctica, una adhesión perfecta no será nunca alcanzada, sin embargo, como quiera se deben desarrollar resinas con un alto nivel de interacción con las fibras. Esta es una tarea muy difícil e importante que debe ser resuelta. En el curso del tiempo, los productores de fibras han hecho un gran esfuerzo para optimizar las formulaciones de los tamaños de las fibras y mejorar las propiedades de adhesión de las fibras a resinas estándar como lo son las resinas epoxi, polyester y vinyl-ester.

Si no existe la adhesión entre las fibras y la matriz, el sistema debe ser considerado indeterminado sin la posibilidad de definir las propiedades mecánicas del compósito. Si un efecto de deslizamiento entre las fibras y la matriz ocurre cuando el compósito se encuentra bajo una carga, el cálculo de las propiedades mecánicas puede resultar impreciso o de difícil interpretación.

Comenzando por esta obvia consideración, en nuestra investigación hemos considerado la adhesión como el factor más importante para un enfoque hacia una nueva generación de matrices compósitas para aplicaciones en la construcción.

\section{EL SISTEMA DE RESINAS IPN}

El sistema Betontex IPN es una novedad absoluta en el campo de las tecnologías de compósitos para la construcción. Éste es un sistema completo que incluye tanto las resinas adhesivas como los refuerzos, estos últimos diseñados y producidos especifícamente para su aplicación con las resinas IPN. El sistema debe ser utilizado exclusivamente mediante la combinación de estos dos componentes (resina y refuerzo), los cuales no pueden ser proporcionados separadamente, como se reporta en la información técnica de la empresa.

\section{ADHESIVOS IPN}

Los adhesivos Betontex IPN son productos de dos componentes a base acuosa y están formados por el componente $\mathrm{A}$, que es una fase líquida de un látex acrílico en dispersión acuosa especialmente formulado y un componente $\mathrm{B}$, que consiste en una composición polimérica dispersa en una fase sólida de un relleno mineral micronizado activo (5). En el proceso de consolidación, el mineral sólido genera microcristales que actúan como un refuerzo de la fase polimérica.

En la figura 2 la morfología especial de la resina IPN consolidada se muestra claramente: 


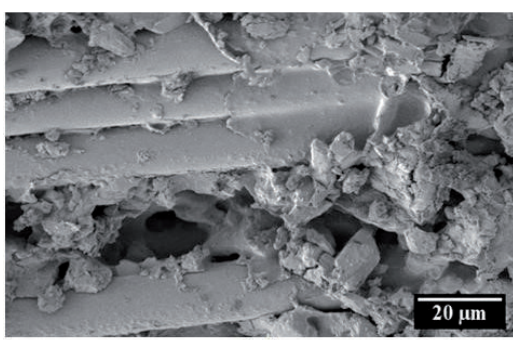

(a)

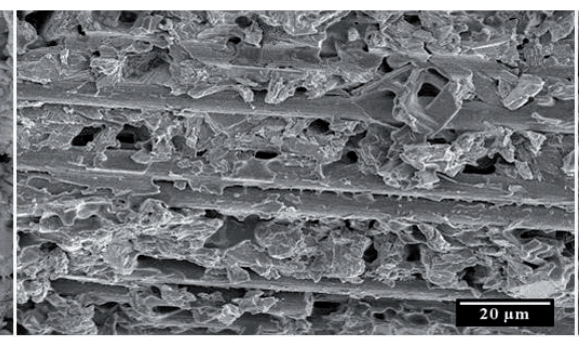

(b)

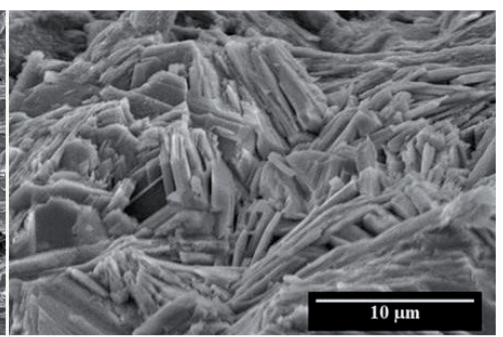

(c)

Figura 2. Micrografías SEM de: - a)- Sección de Vidrio AR y compósito IPN. - b)- Sección de Fibras de carbón y compósito IPN.- c)- Sección de resinas IPN consolidadas mostrando la morfología de los microcristales y un diámetro de cristal de 1-3 mm. ("Enzo FerrariDepartment", University of Modena).

la buena adhesión y el alto nivel de compatibilidad a fibras de vidrio AR (figura 2a) y fibras de carbón (figura 2b) se observa fácilmente; en la figura 2c se puede identificar fácilmente el patrón de microcristales con diámetros aproximados de 1-3 mm conectados mediante la fase polimérica, que está constituida por resina pura consolidada.

En todas las figuras se puede observar una buena dispersión de la micro-porosidad. La morfología de los microcristales de la fase inorgánica activa confiere a la matriz altas propiedades mecánicas y térmicas así como una buena porosidad al vapor de agua (transpiración).

Los adhesivos Betontex IPN pueden ser aplicados con la tecnología FRP para la adhesión directa del refuerzo en concreto, mampostería en ladrillos y piedras o madera en lugar de las resinas epoxi o con la tecnología FRCM como promotor de adhesión en la incorporación directa de capas en morteros a base de cemento o cal.

Las resinas IPN pueden ser proporcionadas con diferentes grados: Primer, Promotor de adhesión, adhesivo impregnador con diferentes valores de viscosidad, Tixoy Grado Masilla. Las propiedades de las resinas IPN se muestran en las tablas 1 y 2.

\section{TABLA 1. PROPIEDADES GENERALES DE LAS RESINAS CONSOLIDADAS BETONTEX IPN}

\begin{tabular}{lr}
\hline PROPIEDADES & VALORES \\
\hline Proporción de los componentes (A/B) & $0,5 / 1$ \\
\hline Densidad $\left(\mathrm{g} / \mathrm{cm}^{3}\right)$ & $\geq 1,5$ \\
\hline Propiedades mecánicas & $80 \%$ de la resina epoxi \\
\hline Adhesión al susbtrato & $\geq 2,5$ \\
\hline (Concreto, Mampostería, Madera) & Delaminación del substrato \\
\hline Resistencia térmica $\left({ }^{\circ} \mathrm{C}\right)$ & 180 \\
\hline Degradación térmica $\left({ }^{\circ} \mathrm{C}\right)$ & 600 \\
\hline Resistencia a la quema $(\mathrm{UNI}) 9177)$ & Clase 1 \\
\hline Adsorción de agua & $\geq 1 \%$ \\
\hline Permeabilidad $(\mathrm{MU})\left({ }^{*}\right)$ & 20 \\
\hline$\left.{ }^{*}\right)$ Medido &
\end{tabular}

(*) Medido en un panel entre dos substratos de cal, espesor total $10 \mathrm{~mm}$.
TABLA 2. PROPIEDADES MECÁNICAS DEL BETONTEX IPN

\begin{tabular}{lr}
\hline PROPIEDADES & VALORES \\
\hline Referido a & Resinas puras \\
\hline Resistencia a la flexión (MPa) & 11 \\
\hline Módulo de flexión (GPa) & 10,9 \\
\hline Resistencia a la tracción (MPa) & 3,7 \\
\hline Módulo Elástico (GPa) & 6,3 \\
\hline Referido a & Compósitos de fibras de carbón (*) \\
\hline Resistencia a la tracción (MPa) & $\geq 2800$ \\
\hline Módulo Elástico (GPa) & 210 \\
\hline Deformación (\%) & $\geq 1,5 \%$ \\
\hline Referido a & Compósitos de fibras de vidrio (*) \\
\hline Resistencia a la tracción (MPa) & $\geq 1000$ \\
\hline Módulo Elástico (GPa) & 70 \\
\hline Deformación (\%) & $\geq 1,8 \%$ \\
\hline
\end{tabular}

$\left.{ }^{*}\right)$ referido a la sección de fibra.

En la tabla 2 las propiedades mecánicas de las resinas puras consolidadas y los compósitos basados en fibras de carbón y vidrio AR son reportadas.

Actualmente estamos llevando a cabo pruebas adicionales encaminadas a la verificación de las propiedades de envejecimiento de las resinas. De acuerdo a una prueba preliminar de envejecimiento de dos años de un laminado IPN de fibras de carbón en un ambiente externo, éste no muestra ningún cambio en estructura y propiedades de datos obtenidos con TCA, DSC y pruebas mecánicas.

REFUERZOS

El sistema de resinas IPN requiere de un diseño específico de la estructura del refuerzo, la cual involucra el uso de tejidos de carbono, de vidrio de zirconio resistentes al alcáli (Betontex Zirconglass Net) y de fibras de aramida (la aplicación de aramida esta siendo actualmente investigada).

La tabla 3 muestra las propiedades de las fibras usadas para los refuerzos que son usados en combinación con las resinas IPN. 
TABLA 3. PROPIEDADES DE LAS FIBRAS USADAS EN LA PREPARACIÓN DE REFUERZOS

\begin{tabular}{lrr}
\hline GRADOS DE FIBRAS / HILO & CARBÓN HT & VIDRIO AR \\
\hline & Tenax UTS & Zirconglass \\
\hline PROPIEDADES / UNIDAD & VALOR & VALOR \\
\hline Peso del Hilo (Tex) & $800-1600$ & $600-2200$ \\
\hline Resistencia a la tracción (MPa) & $\geq 4800$ & $\geq 1400$ \\
\hline Módulo elástico (GPa) & 240 & 74 \\
\hline Deformación máxima (\%) & 2 & 2 \\
\hline Densidad (g/cm3) & 1,78 & 2,5 \\
\hline
\end{tabular}

Las fibras usadas en los tejidos consisten en materias primas de la más alta calidad: las fibras de carbono están certificadas para aplicaciones aeroespaciales y se proporcionan con una garantía del fabricante; las fibras de vidrio AR contienen porcentajes de óxido de zirconio mayor al 19\% - de acuerdo a la normativa UNI EN 15422.

A la fecha, los siguientes tipos de refuerzos han sido optimizados para su uso en combinación con las resinas Betontex IPN: Refuerzo de fibras de carbono:

REFUERZO DE FIBRAS DE CARBONO

- GV160-60 U-IPN: Fibras de carbono unidireccional HT refuerzo de fibras de carbón $\left(160 \mathrm{~g} / \mathrm{m}^{2}\right)$, específicamente modificadas para su uso con la matriz IPN RC170 TH12: Refuerzo de red de fibras de carbono (170 g/ $\mathrm{m}^{2}$ ).

- RC225 TH12 : Refuerzo de red de fibras de carbono $\left(220 \mathrm{~g} / \mathrm{m}^{2}\right)$.

REFUERZO DE FIBRAS DE VIDRIO AR

- RV120-AR: Refuerzo de red de fibras de vidrio AR biaxial - Alambre de vidrio de zirconio $\left(120 \mathrm{~g} / \mathrm{m}^{2}\right)$

- RV220-AR: Refuerzo de red de fibras de vidrio AR biaxial - Alambre de vidrio de zirconio $\left(220 \mathrm{~g} / \mathrm{m}^{2}\right)$

- RV320-AR: Refuerzo de red de fibras de vidrio AR biaxial - Alambre de vidrio de zirconio $\left(320 \mathrm{~g} / \mathrm{m}^{2}\right)$

Estos refuerzos derivan de la tecnología Betontex de termo soldadura por termosellado (6). Los refuerzos de fibras de carbono unidireccionales son modificados mediante la introducción de una fibra de vidrio AR para aumentar el volumen de los tejidos. Las redes son tejidos de lana estandard bidireccionales obtenidos mediante el proceso de termo soldadura anteriormente mencionado, el cual está basado en el proceso de termosellado (6).
La tecnología de termo soldadura permite producir una red en la cual las fibras no son $\mathrm{cu}^{-}$ biertas por plástico, como sucede habitualmente en productos comerciales estándares. Las fibras permanecen libres y los hilos de las fibras pueden ser entonces fácilmente impregnadas por la matriz del compósito, aumentando sus propiedades mecánicas.

\section{APLICACIÓN}

Las resinas IPN pueden ser usadas ya sea en tecnologías de refuerzo FRP o en tecnologías FRCM. Como matriz FRP, el IPN puede ser usado en lugar de las resinas epoxi para fortalecer vigas, columnas, losas, articulaciones, mampostería, bóvedas, arcos, vigas de madera y losas de madera. Existen diferentes grados de resinas disponibles, con diferentes niveles de viscosidad, resinas y grados masilla con propiedades modificadas de tixotropía. La aplicación de la técnica es muy similar a la utilizada para las resinas epoxi con algunas diferencias en la preparación de la superficie del substrato. Además, una notable ventaja de la tecnología IPN consiste en la posibilidad de ser aplicada en superficies húmedas, las cuales no requieren estar perfectamente secas como en el contexto de un compósito basado en epoxi.

Como FRCM, las resinas IPN pueden ser usadas como promotores de adhesión entre las fibras del refuerzo y el mortero. La aplicación de esta tecnología consiste en una primera capa de mortero sobre el susbtrato seguida de la aplicación de una capa de resina IPN sobre el mortero húmedo y la aplicación de la red de refuerzo (carbono o vidrio AR). Finalmente, después de laminar las capas anteriores, se requiere de la aplicación de una segunda capa de mortero para terminar el procedimiento (7).

La cantidad de resinas IPN está limitada a una cantidad de material suficiente para bañar la red de fibras, mientras que el espesor del mortero y la calidad deben ser definidas de acuerdo a las especificaciones de diseño. En cualquier caso, el espesor debe estar entre $3 \mathrm{y}$ $10 \mathrm{~mm}$ con el fin de disminuir el peso soportado por la estructura. Ambos morteros a base de cal o cemento pueden ser usados. Mediante la aplicación de ambos tipos de morteros las principales aplicaciones están limitadas al refuerzo de mampostería, bóvedas, arcos y columnas (7).

En la figura 3a se muestra una representación esquemática de las técnicas FRP y FRCM. 


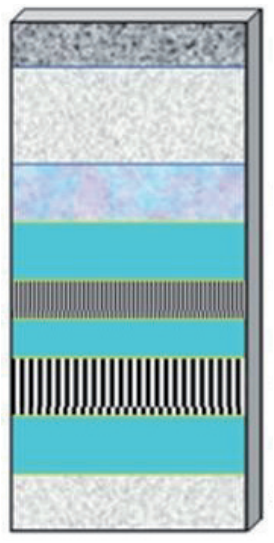

a
Support

$1^{\text {st }}$ layer of mortar

\section{IPN 01 Primer}

$1^{\text {st }}$ layer IPN 02 Adhesive

U-IPN reinforcement $\left(160\right.$ or $300 \mathrm{~g} / \mathrm{m}^{2}$

$2^{\text {nd }}$ - 3rd layer IPN 02 Adhesive

U-IPN reinforcement $\left(160\right.$ or $\left.300 \mathrm{~g} / \mathrm{m}^{2}\right)$

$4^{\text {th }}$ layer IPN 02 Adhesive

$2^{\text {nd }}$ layer or mortar

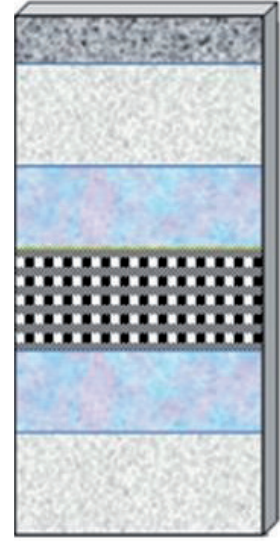

b
Support

$1^{\text {st }}$ layer of mortar

$1^{\text {st }}$ layer IPN 01 Primer

Reinforcement net

$2^{\text {nd }}$ layer IPN 01 Primer

$2^{\text {nd }}$ layer or mortar

Figura 3. Representación esquemática de los esquemas de aplicación FRP (a) y FRCM (b).

\section{EVALUACIÓN DE LAS PROPIEDADES MECÁNICAS} TECNOLOGÍA FRP

Los ensayos de tracción de los compósitos FRP con la matriz IPN en lugar de resinas epoxi fueron llevados a cabo en el Departamento DICAM de la Universidad de Bolonia. El aparato experimental es mostrado en la figura 4a y las dimensiones del especímen de prueba se reportan en la figura 4b. Las probetas fueron obtenidas mediante laminación húmeda layup usando como matriz resinas IPN 01 de bajo grado de viscosidad.

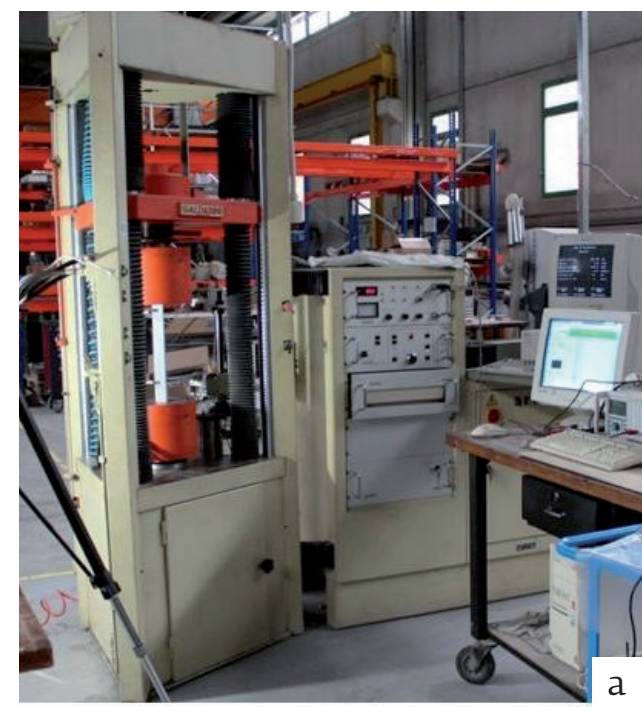

Como se observa en la figura 4b, las probetas tienen dimensiones de $550 \mathrm{~mm}$ de largo, $55 \mathrm{~mm}$ de ancho y un espesor que depende del tejido de refuerzo usado, generalmente entre $1-2 \mathrm{~mm}$ para FRP y $6 \mathrm{~mm}$ para FRCM. Los "tabs" en ambos extremos de la probeta están formados por laminados de fibras de carbono y resina epoxi, aplicados a lo largo de $80 \mathrm{~mm}$ para promover una buena sujeción a la máquina de prueba. Las condiciones de operación están descritas en la normativa ASTM D3039/D3039M, Standard Test Method for Tensile Properties of Polymer Matrix Composite Materials.

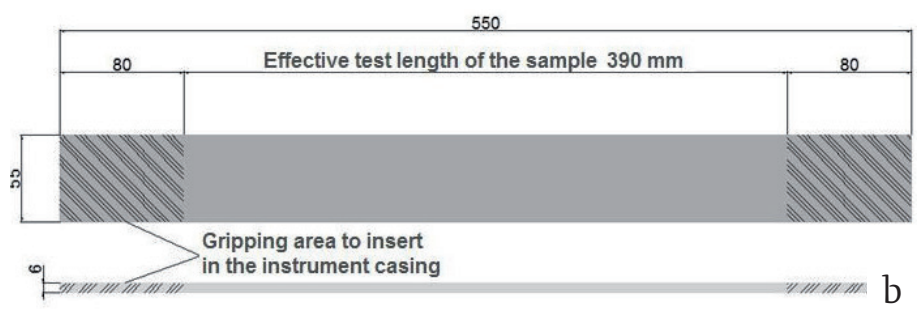

TABLA 4. PROPIEDADES MECÁNICAS DE TRACCIÓN DE LOS LAMINADOS IPN CON REFUERZOS DE CARBONO Y VIDRIO AR. LAS PROPIEDADES SE REFIEREN A LA SECCIÓN DE LA FIBRA EN EL COMPÓSITO. LAS PRUEBAS SE REALIZARON SIGUIENDO LA NORMATIVA ASTM D3039/D3039M-08

\begin{tabular}{|c|c|c|c|c|c|}
\hline REFUERZO & MATRIZ & $\begin{array}{r}\text { SECCIÓN DE LA FIBRA } \\
\left(M^{2}\right)\end{array}$ & $\begin{array}{r}\text { RESISTENCIA A LA TRACCIÓN } \\
\text { PrOMEDIO } \\
\text { (MPA }\end{array}$ & $\begin{array}{r}\text { DEFORMACIÓN } \\
\text { MÁxIMA } \\
(\%)\end{array}$ & $\begin{array}{r}\text { Mó́dUL ELÁSTICO } \\
\text { PROMEDIO } \\
\text { (GPA) }\end{array}$ \\
\hline Red de carbono RC225-TH12 & IPN 01 & 1,79 & 2869 & 1,14 & 226 \\
\hline Red de vidrio de zirconio RV320-AR & IPN 01 & 3,06 & 1013 & 1,59 & 93 \\
\hline
\end{tabular}



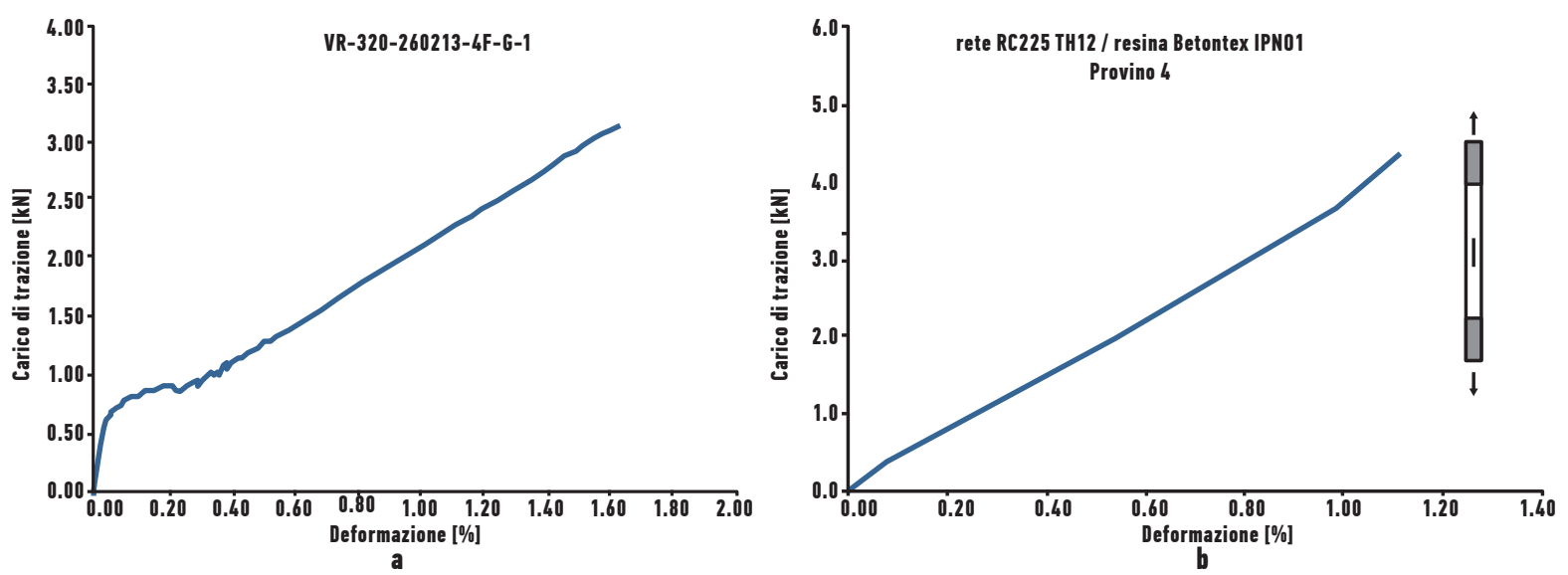

Figura 5. Curvas de carga-alargamiento de los ensayos de tracción en las muestras FRP usando resina IPN como matriz (a) Red de fibras de vidrio AR t RV320-AR (320g/m2); (b) Red de carbono RC225 TH-12 (200g/m²).

La figura 5a y la figura 5b muestran las curvas de carga deformación para los compósitos de carbono y de red de vidrio AR, respectivamente.

Las gráficas muestran la efectividad del IPN para cumplir la función de transferir las cargas al tejido. Esto permite soportar una carga equivalente a la misma que se obtiene usando resinas epoxi.

\section{TECNOLOGÍA FRCM}

Los laminados FRCM fueron preparados usando ya sea morteros de cal y/o morteros de cemento. Considerando los campos de aplicación, el compósito FRCM a base de mortero de cal parece ser la tecnología más interesante para ser usada para el refuerzo de construcciones de mampostería, las cuales representan la tecnología preferida para la recuperación de una gran parte del patrimonio histórico en Italia y alrededor del mundo.

Se debe tener mucho cuidado en la preparación de la probeta para el ensayo de tracción ya que la enorme fragilidad del mortero de cal se hace evidente para espesores pequeños.

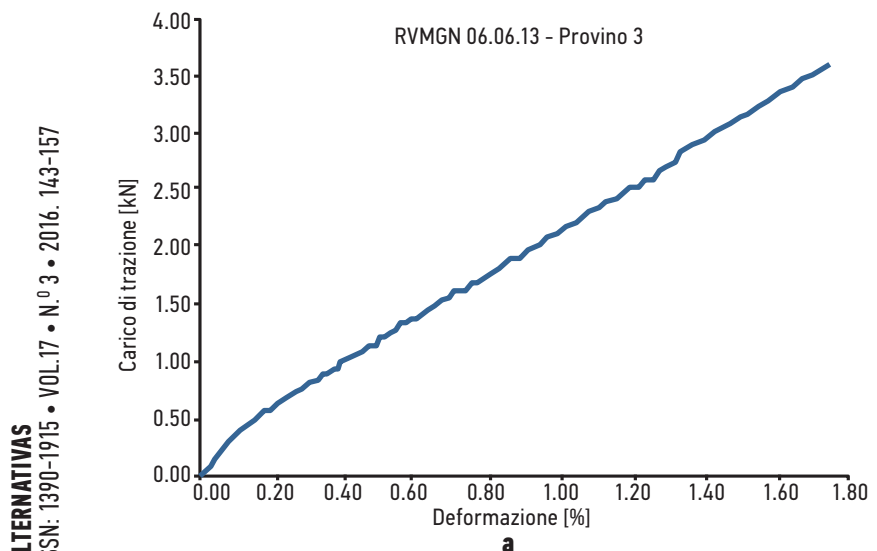

Figura 6. Ensayo de tracción del mortero de cal FRCM reforzado con la red RV320: a) usando el promotor de adhseión IPN y b) sin promotor de adhesión.
De acuerdo con los mecanismos de fractura, es obvio que capas más espesas de mortero afectarán el comportamiento del material durante el ensayo de tracción y en general durante la carga.

La posibilidad de usar promotores de adhesión, como la resina IPN01, es de gran ayuda durante el proceso de preparación de la muestra, pero debido al bajo espesor del mortero, nosotros dedicamos una gran parte de esta investigación a la optimización de este procedimiento.

Generalmente, las probetas están formadas por $3 \mathrm{~mm}$ de capa de mortero, una capa del tejido de refuerzo previmente bañado con el promotor de adhesión IPN y una segunda capa de $3 \mathrm{~mm}$ del mortero. El espesor total de la probeta es de $6 \mathrm{~mm}$. El consumo total promedio del promotor de adhesión está comprendido entre $500-800 \mathrm{~g} / \mathrm{m}^{2}$, en función del peso del tejido. Los especímenes FRCM tienen las mismas dimensiones que las muestras FRP, como se reporta en la figura 4b.

En las figuras 6 y 7, los ensayos de tracción de las probetas de cal con las redes de vidrio

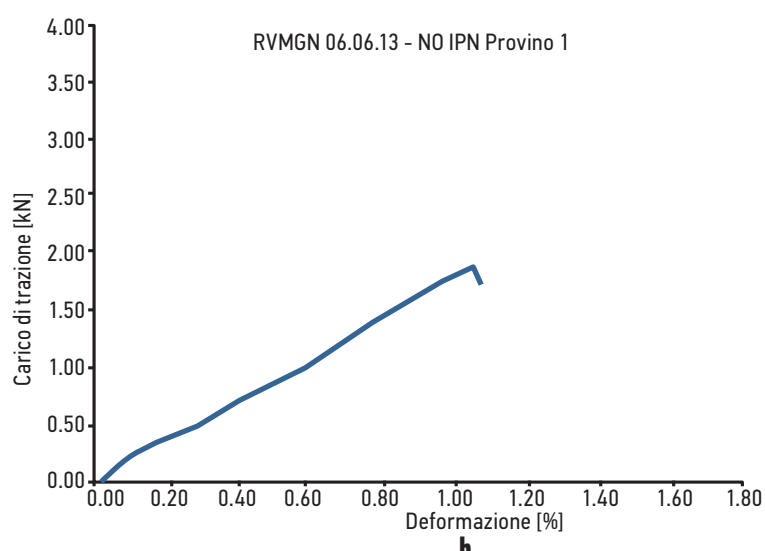



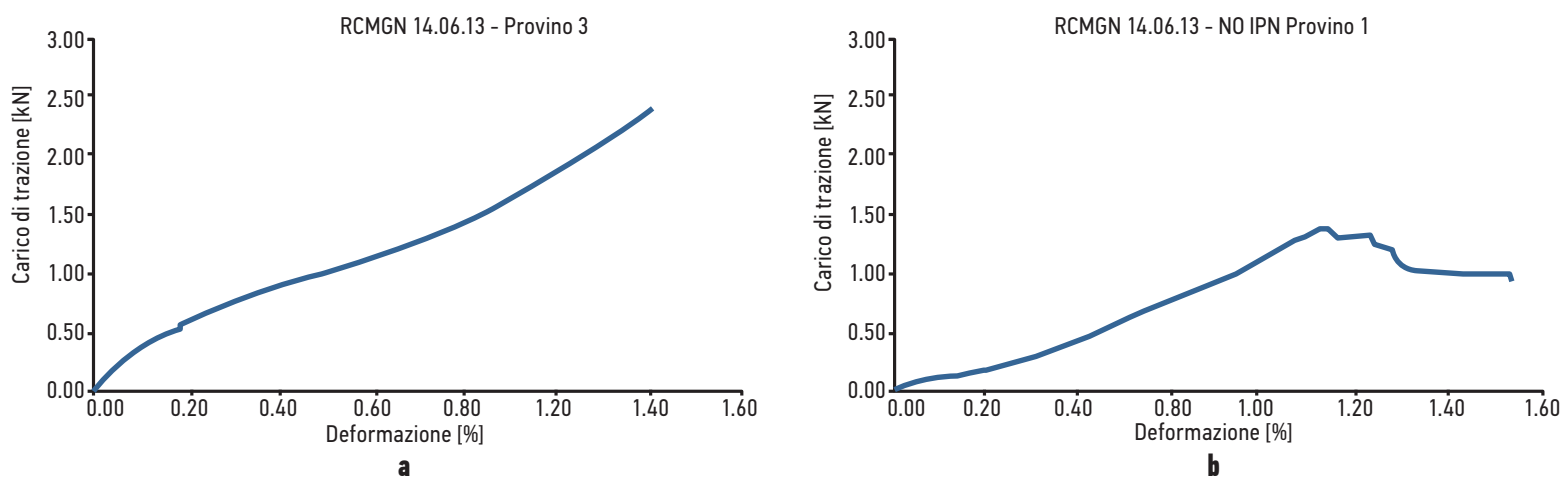

Figura 7. Ensayo de tracción del mortero de cal FRCM reforzado con la red de carbón RC170 TH12: a) usando el promotor de adhseión IPN y b) sin promotor de adhesión.

\begin{tabular}{|c|c|c|c|c|c|}
\hline REFUERZO & MATRIZ & $\begin{array}{r}\text { SECCIÓN DE LA FIBRA } \\
\left(\text { (MM²) }^{2}\right.\end{array}$ & $\begin{array}{r}\text { RESISTENCIA A LA TRACCIÓN } \\
\text { PROMEDIO } \\
\text { (MPA }\end{array}$ & $\begin{array}{r}\text { DEFORMACIÓN } \\
\text { MÁxIMA } \\
(\%)\end{array}$ & $\begin{array}{r}\text { MódULO ELÁSTICO } \\
\text { PROMEDIO } \\
\text { (GPA) }\end{array}$ \\
\hline Red de carbono RC225-TH12 & IPN 01 & 1,79 & 2869 & 1,14 & 226 \\
\hline Red de vidrio de zirconio RV320-AR & IPN 01 & 3,06 & 1013 & 1,59 & 93 \\
\hline
\end{tabular}

TABLA 5. RESULTADOS DE LOS ENSAYOS MONOTÓNOS DE TRACCIÓN DEL FRCM A BASE DE MORTERO DE CAL

\begin{tabular}{|c|c|c|c|c|c|}
\hline REFUERZO & $\begin{array}{l}\text { PROMOTOR DE } \\
\text { ADHESIÓN IPN }\end{array}$ & $\begin{array}{r}\text { CAROA } \\
\text { MÁXIMA } \\
\text { (KN) }\end{array}$ & $\begin{array}{r}\text { RESISTENCIA A } \\
\text { TRACCIÓN } \\
\text { (MPA) }\end{array}$ & $\begin{array}{r}\text { MÁXIMO } \\
\text { ALARGAMIENTO (\%) }\end{array}$ & MODO DE FALLO \\
\hline Red de fibra de vidrio RV320-AR & Sí & 3,6 & 1190 & 1,7 & Rotura de las fibras \\
\hline Red de fibra de vidrio RV320-AR & No & 1,87 & 615 & 1 & Deslizamiento de las fibras \\
\hline Red de carbono RC170TH12 & Sí & 6,3 & 2360 & 1,3 & Rotura de las fibras \\
\hline Red de carbono RC170TH12 & No & 2,76 & 1034 & 0,9 & Deslizamiento de las fibras \\
\hline
\end{tabular}

AR y carbono, aplicados con y sin promotor de adhesión IPN, son mostradas usando la misma escala en los ejes $\mathrm{x}-\mathrm{y}$.

Debido a la naturaleza frágil de las matrices del mortero y, para aumentar la eficiencia de adhesión en el comportamiento mecánico de los materiales (considerando las aplicaciones como refuerzos antisísmicos) deacuerdo a las indicaciones de Savoia (8), los ensayos

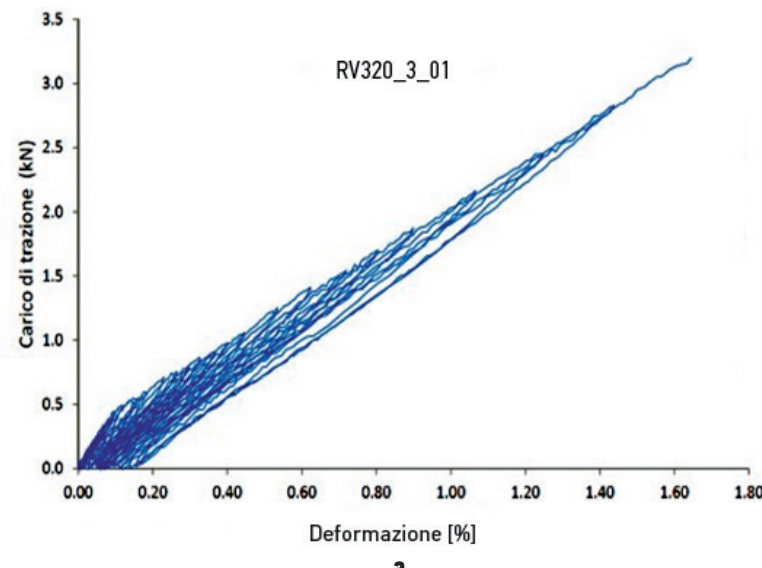

de tracción fueron repetidos en condiciones de carga cíclica. Antes de alcanzar la carga máxima y la ruptura de la muestra, un número importante de ciclos de carga fueron aplicados, alcanzando hasta 28 ciclos. En las figuras 8 y 9 , las pruebas de carga cíclica en los espcímines de cal con red de vidrio AR y red de carbono, respectivamente, con y sin promotor de adhesión IPN, son reportados.

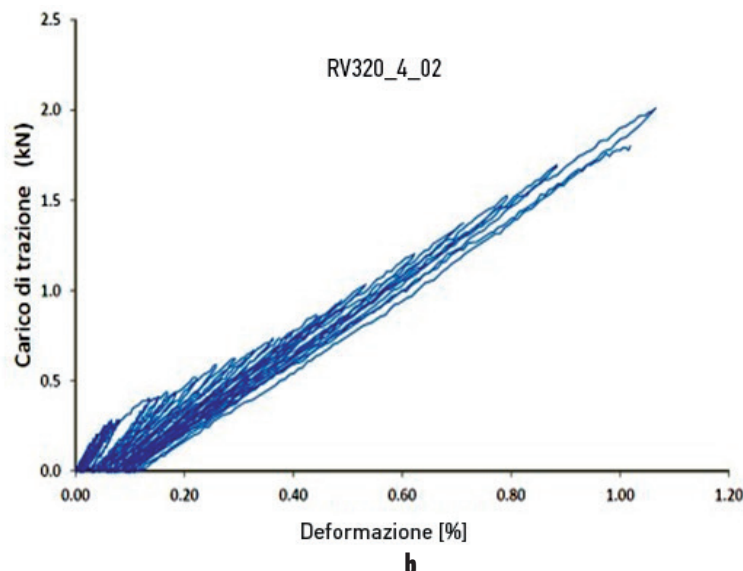

Figura 8. Ensayos de tracción cíclicos del mortero de cal FRCM reforzado con red RV320 - AR : a) usando promotor de adhesión IPN y b) sin promotor de adhesión (Prestar atención a las diferentes escalas de los ejes x y y) (8). 

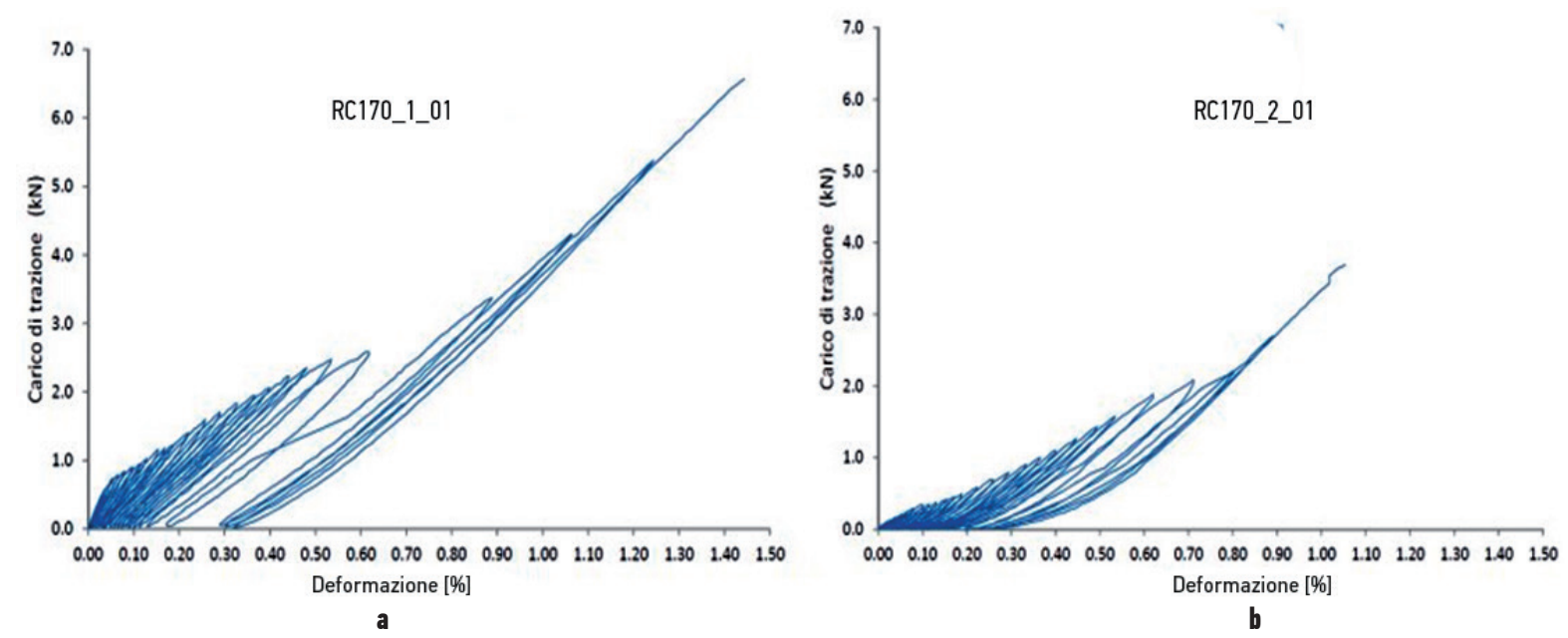

Figura 9. Ensayos de tracción cíclicos del mortero de cal FRCM reforzado con red RC170 TH12: a) usando promotor de adhesión IPN y b) sin promotor de adhesión (8).

TABLA 6. RESULTADOS DE LOS ENSAYOS DE TRACCIÓN CÍCLICOS DEL FRCM A BASE DE MORTERO DE CAL

\begin{tabular}{|c|c|c|c|c|c|}
\hline REFUERZO & $\begin{array}{l}\text { PROMOTOR DE ADHESIÓN } \\
\text { IPN }\end{array}$ & $\begin{array}{l}\text { CARGA MÁXIMA } \\
\text { (KN) }\end{array}$ & $\begin{array}{l}\text { RESISTENCIA A LA } \\
\text { TRACCIÓN } \\
\text { (MPA) }\end{array}$ & $\begin{array}{l}\text { ALARGAMIENTO } \\
\text { MÁXIMO } \\
(\%)\end{array}$ & MODO DE FALLO \\
\hline Red de fibra de vidrio RV320-AR & Sí & 3,3 & 1080 & 1,64 & Ruptura de las fibras \\
\hline Red de fibra de vidrio RV320-AR & No & 2,1 & 680 & 1,12 & Deslizamiento de las fibras \\
\hline Red de carbón RC170TH12 & Sí & 6,7 & 2500 & 1,35 & Ruptura de las fibras \\
\hline Red de carbón RC170TH12 & No & 3,7 & 1385 & 1,05 & Deslizamiento de las fibras \\
\hline
\end{tabular}

En la tabla 6, los resultados de los ensayos de tracción cíclicos de los FRCM a base de mortero de cal son resumidos. Se debe hacer énfasis en el modo de fallo, con cambios evidentes estrechamente relacionados con el patrón de desarrollo de grietas durante la prueba, modificado por la influencia del promotor de adhesión IPN.

Analizando los datos reportados en la tablas 5 y 6 , es claro que pruebas dinámicas de 28 ciclos proporcionan resultados muy similares a aquellos obtenidos con las pruebas monótonas. Además, el efecto del promotor de adhesión afecta el mecanismo de ruptura de los especímenes, los cuales son completamente diferentes con y sin promotor.

Cuando el IPN es usado como promotor de adhesión, la falla se debe a la ruptura de las fibras, aún en el caso del refuerzo de carbono. Por el contrario, sin las resinas IPN la ruptura ocurre prematuramente mediante el efecto del deslizamiento de las fibras conjugado con la separación del mortero, como consecuencia de la falta de adhesión. Además, los valores máximos y la deformación son gobernados por la adhesión entre las fibras y la matriz del mortero, lo que impide aprovechar las potencialidades reales de las fibras.

Se debe hacer enfásis en que el número de ciclos se reduce considerablemente en ausencia de la adhesión; ésta es una desvetaja crítica en presencia de efectos sísmicos. Cuando se aplica un promotor de adhesión como la resina IPN, el espécimen permanece perfectamente eficiente hasta la ruptura, sin ningún daño después de ser expuesto a un gran número de cíclos y con una recuperación completa de las deformaciones cuando la carga es reducida. Este comportamiento es una buena demostración de la eficiencia de los refuerzos en caso de fenómenos sísmicos.

\section{APLICACIONES EN PRUEBAS DE LABORATORIO DE MAMPOSTERÍA}

La aplicación de las resinas IPN en mampostería fue evaluada a nivel laboratorio en columnas y paredes con diferentes configuraciones de refuerzos para aplicación FRP y FRCM con mortero de cal. La figura 10 muestra las pruebas de laboratorio en el departamento CIRI de la Universidad de Bolonia de una columna en ladrillos evaluada en compresión y una pared de ladrillos evaluada en ensayo de corte (prueba de compresión diagonal), respectivamente (8).

Sin entrar en detalles en esta investigación, la cual sería el objeto de un artículo específico, se llegó a la conclusión de que los resultados obtenidos con las resinas IPN son perfectamente similares y en algunos casos superiores a aquellos obtenidos con las resinas epoxi. 


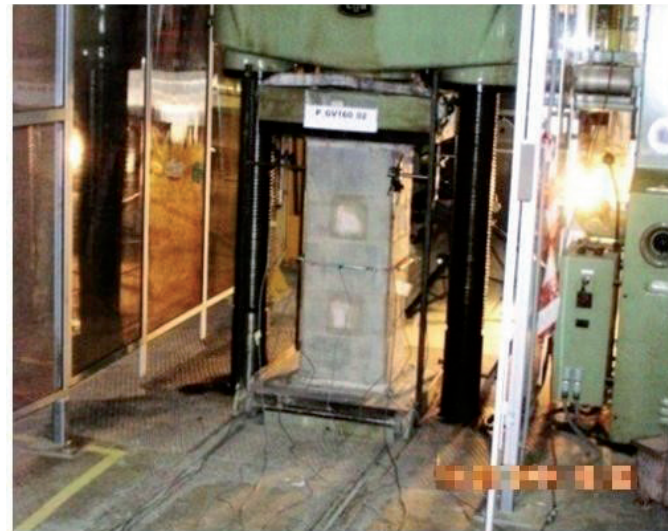

(a)

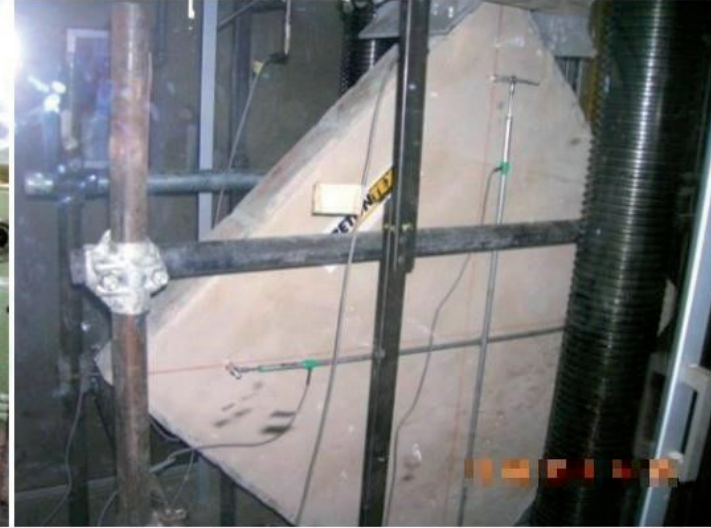

(b)

Figura 10. Pruebas de laboratorio de columnas y paredes de ladrillo, reforzadas con la tecnología FRCM usando resinas a base acuosa IPN y mortero de cal (Universidad de Bolonia- Departamento CIRI).

\section{APLICACIÓN SOBRE SUSBTRATOS DE CONCRETO}

Un importante campo para la aplicación, investigación y desarrollo de las resinas IPN dentro de las tecnologías FRP involucra el reforzamiento de estructuras de concreto como vigas, pilares, paredes y losas. La experiencia ha demostrado la efectividad de los refuerzos de fibras largas en términos de respuesta a la flexión, cizallamiento y arriostramento (bracing en inglés) y la resistencia de la viga debe ser aumentada de manera considerable a través de estructuras de actualización contra eventos sísmicos.

Una campaña de investigación de la aplicación y evaluación de compósitos a base de la resina IPN en vigas de concreto fue llevada a cabo en el Departamento DICAM de la Universidad de Bolonia (9). En esta campaña se tenían dos objetivos específicos: el primero consistía en el estudio de comportamiento de flexión de la viga, el segundo involucraba el comportamiento de cizallamiento. Además se realizó una comparación con vigas con resinas epoxi precedentemente evaluadas (1-2).

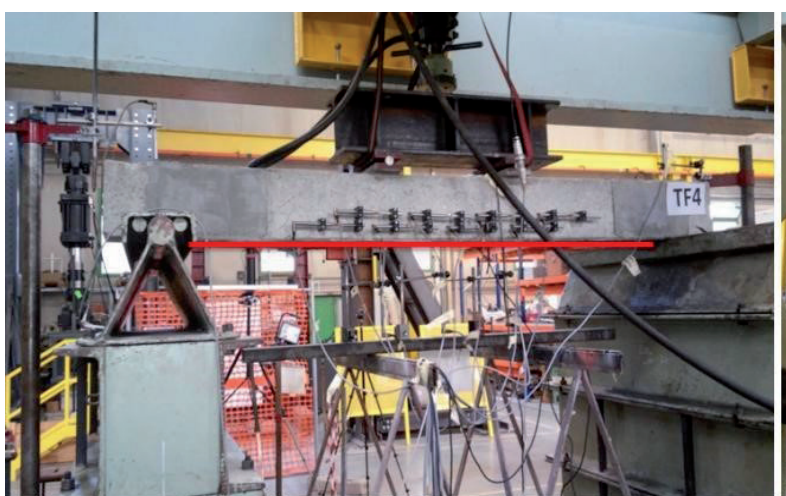

(a)
Para completar la serie de pruebas, verificamos la potencialidad real de dispositivos encaminados a evitar mecanismos de delaminación, como cintas en forma de "U" o sistemas de anclaje Ardfix. Los conectores Ardfix son descritos en las referencia (10) y están compuestos de varillas de carbono pultrusionadas (pultruted) insertadas en la viga, junto con dos cintas de carbono unidireccional, el cual también provee un refuerzo adicional de cizallamiento como una ligadura. Todos los especímenes fueron evaluados mediante una prueba de flexión a cuatro puntos. En la figura 11 se muestra el aparato para la prueba.

La figura 11a representa una viga reforzada con tejido unidireccional GV160-60 U-IPN (4 capas sobrepuestas) con el fin de rellenar la brecha en términos de carga de flexión, además de los dispositivos con forma de "U" para retrasar la activación de los fenómenos de desunión de los finales. La figura 11b representa una viga destinada a superar las cargas de alta cizalla en la medida que sus soportes de acero ofrecen baja resistencia.

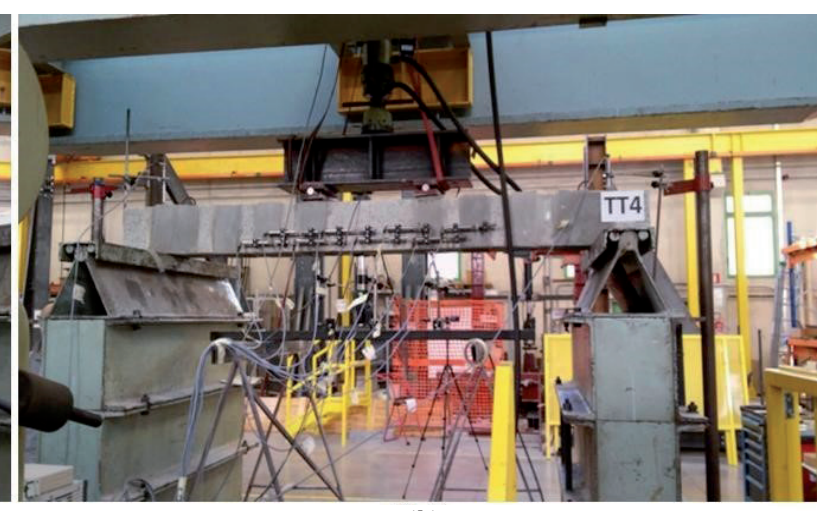

(b)

Figura 11. Aparato experimental para las pruebas de flexión (Prueba 4PB) (a) un viga reforzada en términos de resistencia a la flexión (b) una viga reforzada en términos de resistencia al cizallamiento. 
Resumiendo los resultados de este estudio específico, se evidencia una vez más que las resinas IPN usadas como matriz FRP alcanzan los rendimientos mecánicos obtenidos con las resinas epoxi, como reportado en la figura 12 y la tabla 7. La figura 12 muestra que el uso de la resina IPN como matriz permite a los especímenes fallar al mismo grado de carga que los espcímenes con el adhesivo epoxi.

En la figura 12 el comportamiento de las pruebas de carga - desviación de las siguientes vigas son reportados:T0 Viga no refozada;. TFB se refiere a la viga estándar reforzada usando un refuerzo FRP a base epoxi sin dispositivos anti-desunión (1-2); TFP se refiere a una viga usando refuerzo FRP a base epoxi con dispositivo anti-desunión (1-2); TF1 se refiere a una viga reforzada con el refuerzo IPN aplicado sin dispositivos anti-deusnión y TF2 una viga reforzada con refuerzo IPN aplicado con dispositivos antidesunión. Todos las vigas reforzadas presentan la misma sección de fibra de carbón.
En la tabla 7, los resultados pueden ser fácilmente comparados y se puede confirmar la equivalencia entre los laminados FRP usando resinas epoxi y sus análogos con la nueva resina a base acuosa IPN: los rangos de los valores son absolutamente comparables.

\section{EJEMPLOS DE CASOS REALES DE REHABILITACIÓN ESTRUCTURAL}

Junto a los esfuerzos ficalizados en la optimización de las tecnologías IPN, una experiencia de aplicación importante en edificios se llevó a cabo principalmente en rehabilitación estructural de edificios históricos.

En estos casos el diseño de los trabajos de restauración debe seguir obligaciones rígidas, ya sea en términos de procedimientos y en la selección de materiales y tecnología. Primero que nada el uso de resinas epoxi es usualmente desaconsejado debido a su baja repirabilidad al agua y humedad. La retención de agua dentro de los paneles de mampostería puede causar daños

\section{Flexural tests: load vs deflection graph}

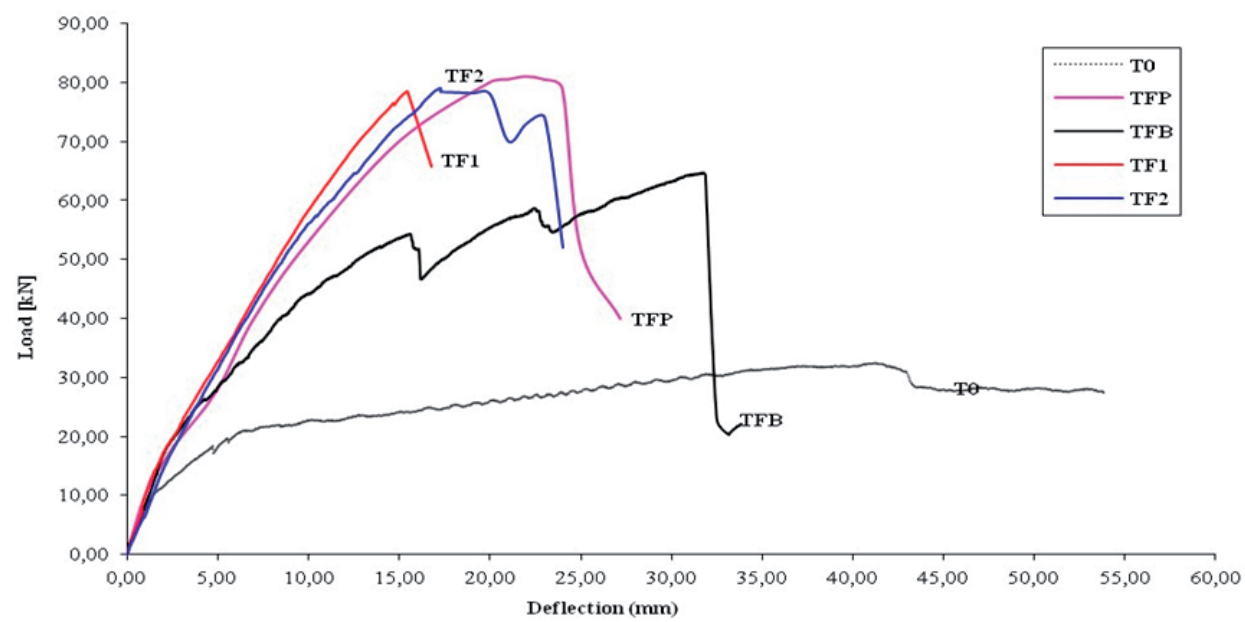

Figura 12. Pruebas de flexión comparativas en vigas de concreto.

TABLA 7. COMPARACIÓN ENTRE CAMPAÑAS EXPERIMENTALES PRECEDENTES (REFUERZOS DE RESINAS EPOXI) Y LA CAMPAÑA IPN EN TÉRMINOS DE CARGA MÁXIMA (CONSIDERE LA CARGA TOTAL APLICADA POR EL PISTÓN HIDRÁULICO)

\begin{tabular}{|c|c|c|c|c|c|c|}
\hline ID & $\begin{array}{l}\text { REFUERZO } \\
\text { OBJETIVO }\end{array}$ & MATRIZ & DISPOSITIVO & CARGA (KN) & MODO DE FALLA & AUTORES \\
\hline TO & Ninguno & Ninguno & Ninguno & $(22-33)^{*}$ & Grietas marcadas & PoluzziBartoli \\
\hline TF1 TFB & $\begin{array}{l}\text { Flexión } \\
\text { Flexión }\end{array}$ & IPN $($ Epoxi)* & Ninguno & $\begin{array}{l}74-92 \\
(60-65)^{*}\end{array}$ & $\begin{array}{l}\text { Desunión con separación del } \\
\text { concreto }\end{array}$ & $\begin{array}{l}\text { Guiduzzi et al. } \\
\text { Poluzzi-Bartoli }\end{array}$ \\
\hline $\begin{array}{l}\text { TF2 } \\
\text { TFP }\end{array}$ & $\begin{array}{l}\text { Flexión } \\
\text { Flexión }\end{array}$ & IPN $(\text { Epoxi) })^{*}$ & Soportes terminales en forma de "U" & $\begin{array}{l}80-92 \\
(88-98)^{*}\end{array}$ & $\begin{array}{l}\text { Colapso de cizalla en las } \\
\text { terminaciones }\end{array}$ & $\begin{array}{l}\text { Guiduzzi et al. } \\
\text { Poluzzi- Bartoli }\end{array}$ \\
\hline $\begin{array}{l}\text { TF3 } \\
\text { TPB }\end{array}$ & Flexión & IPN (Epoxi) & $\begin{array}{l}\text { Conectores Ardfix } \\
(\Delta=200 \mathrm{~mm})^{* * *}\end{array}$ & $\begin{array}{l}80-82 \\
(81-85)_{* *}\end{array}$ & $\begin{array}{l}\text { Colapso de cizalla en las } \\
\text { terminaciones }\end{array}$ & $\begin{array}{l}\text { Guiduzzi et al. } \\
\text { Bartoli }\end{array}$ \\
\hline
\end{tabular}

$\left({ }^{*}\right)$ Rango obtenido en pruebas precedentes usando matriz epoxi, Poluzzi [1] - Bartoli [2] .

$\left.{ }^{(* *}\right)$ Rango obtenido en pruebas precedentes usando matriz epoxi, Bartoli [2] $\left.{ }^{* * *}\right)$ Distancia entre los conectores Ardfix Connectors dentro las vigas. 


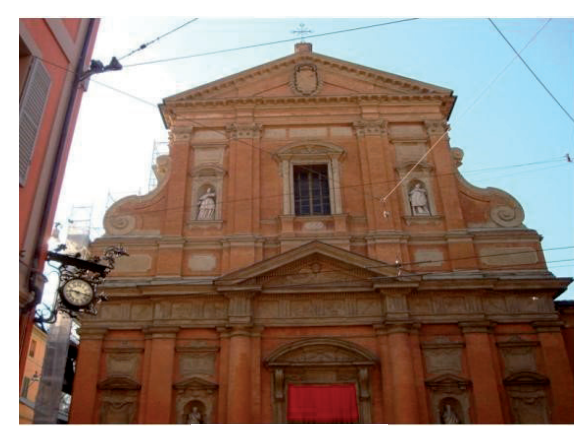

(a)

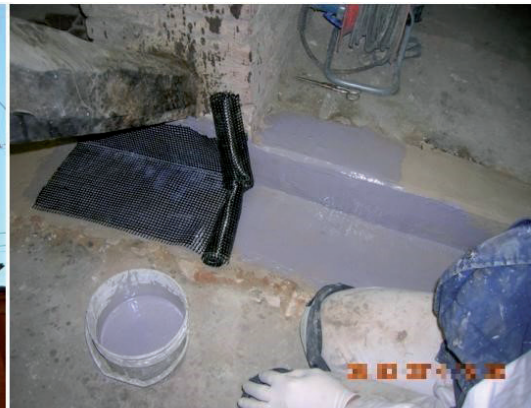

(b)

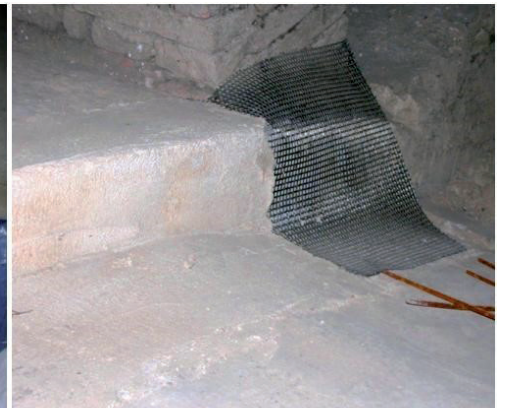

(c)

Figura 13. Trabajos de rehabilitación usando la tecnología FRCM, en una bóveda de mampostería dañada por la deformación de la estructura. (a) Fachada externa de la Iglesia de San Paolo Maggiore en Bolonia. (b) Laminación de la red de carbón con el promotor de adhesión IPN sobre la primera capa del mortero de cal. (c) Trabajo terminado. (Diseño estructural del Prof. R. Poluzzi).

y desprendimiento de partes creando una situación de peligro en presencia de frescos valiosos.

Un ejemplo es el refuerzo de bóvedas de mampostería en la Iglesia San Paolo Maggiore en el centro de la ciudad de Bolonia (figura 13a), las cuales fueron reforzadas usando un mortero de cal y el promotor de adhesión IPN. Esta tecnología permite aumentar las propiedades mecánicas del composito, mejorando la adhesión interlaminar y mantener sin cambios la respirabilidad natural de la cal (proporción total del polímero menos del 5\% - como prescrito). Los principales pasos de la aplicación se reportan en las figuras $13 \mathrm{~b}-\mathrm{c}$.
Otra propiedad útil de las resinas a base acuosa IPN consiste en su gran resistencia térmica y alta resistencia a la quema. De hecho, de acuerdo a la normativa UNI 9177, las resinas IPN pueden ser aplicadas en contextos sensibles a problemas con el fuego, como en teatros. En la figura 14, la aplicación de los sistemas de refuerzo IPN en el refuerzo antisísmico del Teatro de la Ciudad de Carpi (Modena) es reportado.

Además, superados los problemas de las resinas epoxi en presencia de agua y humedad durante la aplicación, el IPN puede ser aplicado sobre superficies húmedas y bañadas, siendo frecuentemente la única solución para la re-

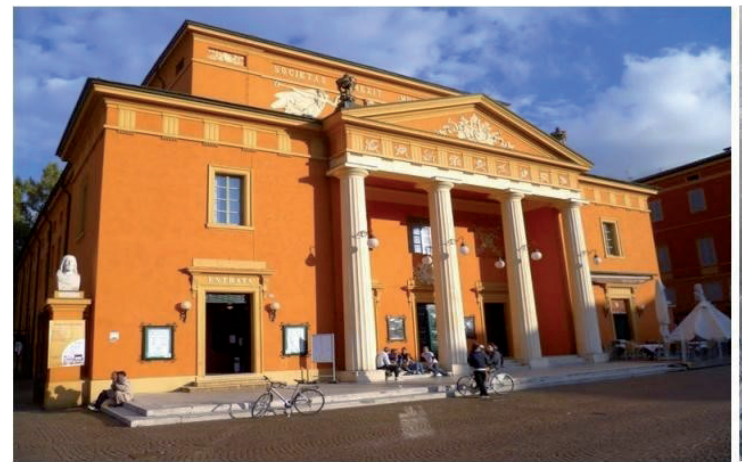

(a)

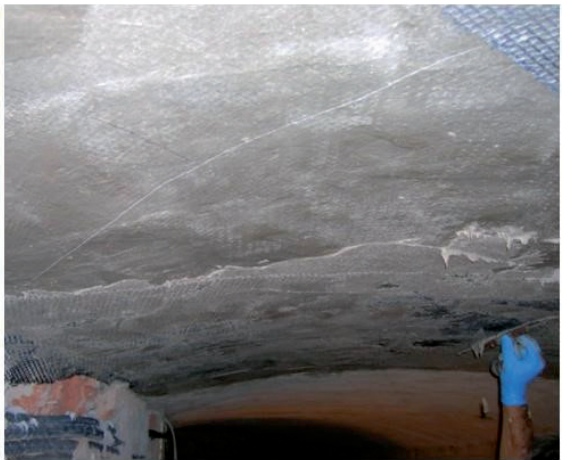

(b)

Figure 14. Reforzamiento antisísmico usando la tecnología FRCM y el sistema IPN en bóvedas de mampostería. (a) Fachada externa del Teatro de la Ciudad ed Carpi, Carpi - Modena. (b) Aplicación de las últimas capas del mortero de cal (Aplicación: Resin Proget Srl -ltaly).

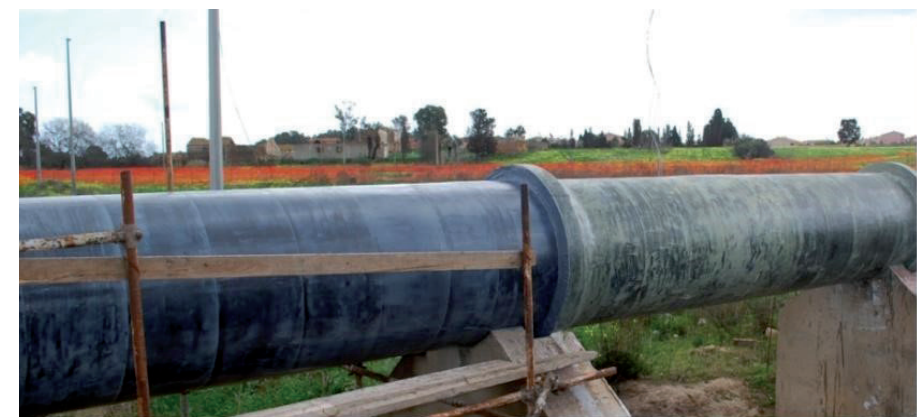

(a)

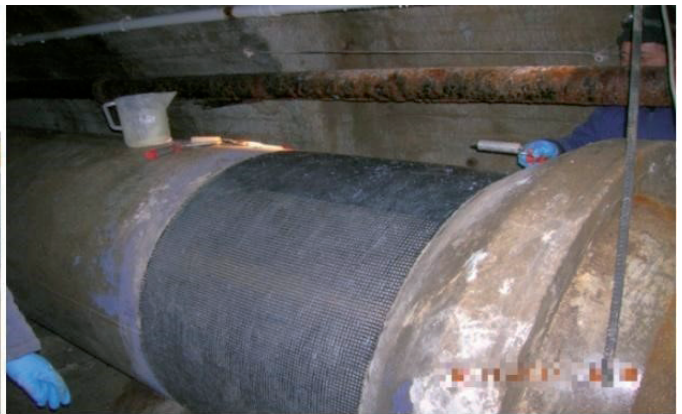

(b)

Figure 15. Aplicación de resinas IPN en la rehabilitación y refuerzo de infraestructuras hidráulicas de acueductos de concreto, en combinación con una resina epoxi. (a) Acueducto industrial de concreto del Río Flumentosa - Sardinia (b) Acueductos civiles de Lucania - Sur de Italia - Potenza. (Diseño y tecnologia Ing. Gianluca Ussia y Prof. L.A.Credali). 

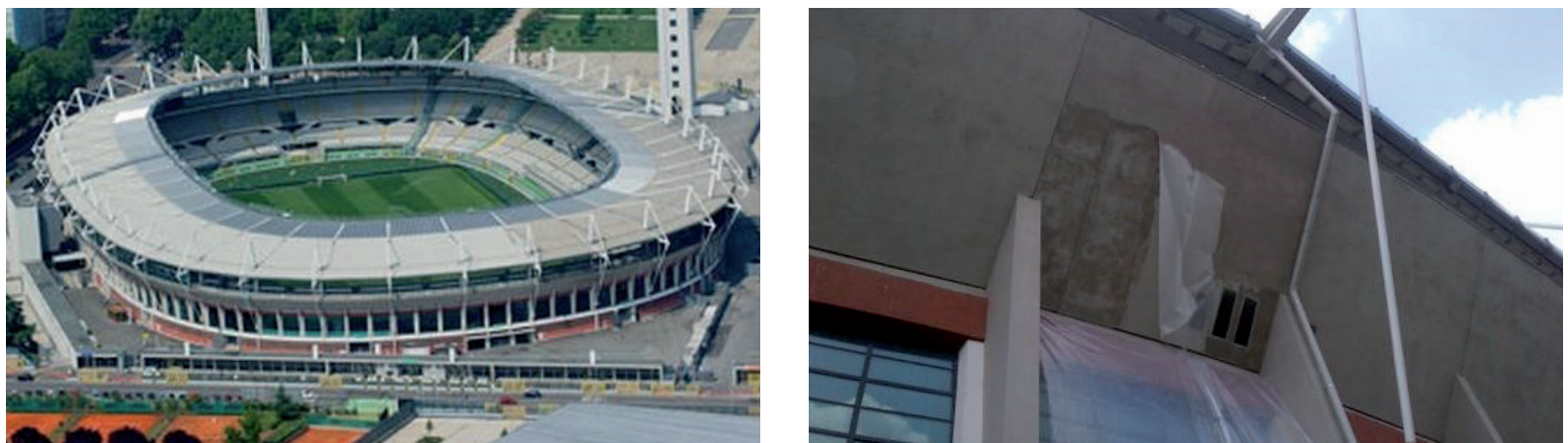

Figura 16. Applicación de la tecnología FRCM para asegurar páneles de concreto del Estadio Olímpico (Turín). (a) Vista aérea del estadio (fuente: La Stampa). (b) Aplicación de las últimas capas del mortero de cal sobre las fibras y el refuerzo IPN. (Diseño estructural Ing. Gianluca Ussia).

paración de infraestructuras hidráulicas como tubos de presión o estructuras subterráneas, donde los soportes pueden ser regularmente afectados por agua.

Un ejemplo es la reparación de acueductos de cemento, los cuales lixivian agua debido a la porosidad del concreto. En la figura 15 dos ejemplos son reportados, en los cuales un correcto diseño usando ambas tecnologías de laminación IPN y epoxi permitieron reparar exitosamente las propiedades mecánicas originales de los acueductos, evitando pérdidas de agua.

Para concluir este reporte, notése que los compósitos FRCM basados en IPN han sido utilizados para asegurar paneles modulares prefabricados de concreto del Estadio Olímpico de Turín, como se muestra en la figura 16, con especial énfasis de la aplicación final del mortero de cal (figura 16b).

CONCLUSIONES

La investigación de Ardea reportada en este artículo ha envidenciado las importantes propiedades y el gran potencial del nuevo sistema de resinas a base acuosa Betontex IPN ${ }^{\circledR}$, que deben ser consideradas una revolución en las tecnologías de compósitos para construcciones. Las características significativas del sistema IPN para ser usadas en las tecnologías FRP y FRCM son el primer y más importante aspecto. En ambos casos, usado como resina matriz en FRP o usado como promotor de adhesión en FRCM, las resinas IPN pueden superar muchos problemas relacionados con estas dos tecnologías.

En aplicaciones FRP, los compósitos IPN comparados con las resinas epoxi dan resultados muy similares con las ventajas decritas en este reporte: no se queman, no poseen temperatura de transición vítrea de segundo orden, tienen alta resistencia a la temperatura, permeabili- dad a la humedad y se pueden aplicacar sobre superficies húmedas.

En las aplicaciones FRCM donde los sistemas Betontex IPN son usados como promotores de adhesión, los resultados de la investigación demostraron un gran mejoramiento de las propiedades mecánicas, las cuales representan una característica importante de la tecnología, como por ejemplo el mortero de cal reforzado con fibras que puede alcanzar propiedades de tracción muy cercanas al $80 \%$ de los laminados epoxi.

En nuestra investigación, hemos focalizado especial atención a la adhesión entre la fibra y la matriz y a un apropiado sistema de evaluación para evidenciar sus efectos en las propiedades de los materiales

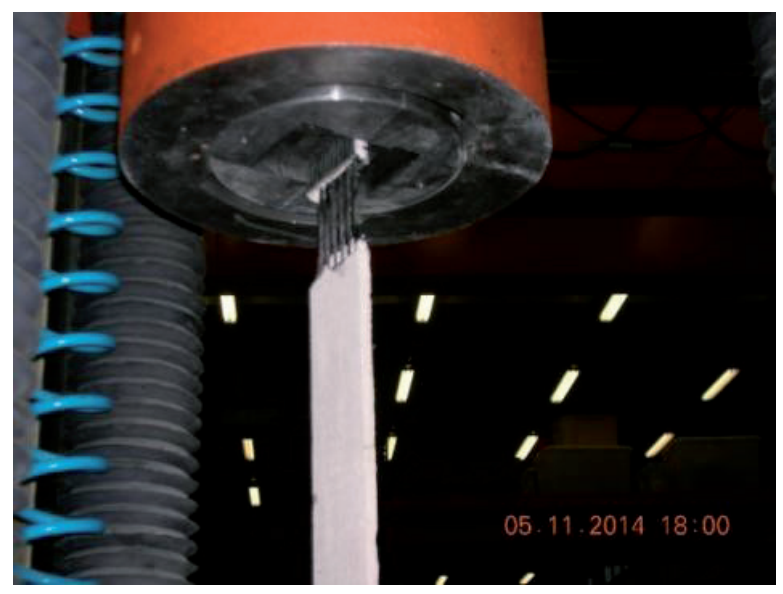

Figura 17. Ensayo de tracción de la muestra de cal FRCM sin el promotor de adhesión de las fibras, el deslizamiento de las fibras comienza antes de la formación de grietas.

Un extensivo programa de evaluación fue desarrollado. Los ensayos de tracción cíclicos parecen ser muy apropiados para esta investigación y se encontró que pruebas cíclicas pueden discriminar de manera precisa el cómo se comportan los materiales bajo estrés. El deslizamiento de fibras, con respecto a las matrices (figura 17) afecta la estabilidad del material y la adhesión del substrato a la fibra con la reducción del 
rendimiento del refuerzo. La adhesión de las fibras a las matrices (cal o cemento) inducidas por el IPN aporta propiedades de tracción hasta alcanzar la ruptura de las fibras. Esta es una condición que permite evaluar y predecir las propiedades mecánicas de los compósitos de acuerdo a la ecuación [1].

Las pruebas de adhesión en las muestras FRCM (figura 17) con y sin el promotor de adhesión evidenciaron claramente el rol de las resinas IPN. Cuando se forman las grietas primero, sin IPN, un desprendimiento de la matriz ocurre y el deslizamiento de las fibras comienzan a alcanzar la ruptura máxima de la muestra.

En esta situación, las propiedades mecánicas del FRCM no están influenciadas por las propiedades de la fibra y serán dependientes sólo de la resistencia a la tracción del mortero. Esta consideración es indicativa de que cualquier tipo de fibras pueden ser usadas sin afectar las propiedades mecánicas del material, las cuales serán gobernadas sólo por la resistencia del mortero. Para mejorar las propiedades mecánicas del FRCM en absencia del promotor de adhesión de las fibras, la única solución que puede es aplicar una modificación importante al polímero del mortero, con la introducción de al menos el $20 \%$ de una fase polimérica en la matriz.

Cuando el IPN es usado como promotor de adhesión, la carga en la ruptura alcanza la resistencia a la tracción de las fibras como se muestra en la figura 18.

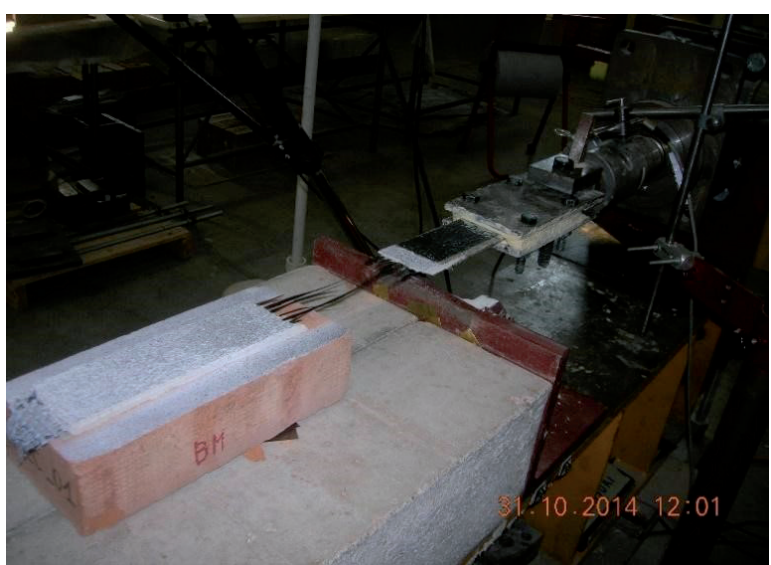

Figura 18. Prueba de resistencia al cizallamiento del FRCM basado en mortero de cal y fibra de carbono unidireccional, usando el promotor de adhesión IPN, la ruptura de la fibra de carbón es claramente evidenciada (8).

Los promotores de adhesión IPN, como consecuencia de la adhesión entre la fibra y la matriz, permiten el aprovechamiento de las propiedades mecánicas de las fibras, las cuales soportan las cargas.
Sin el deslizamiento entre las fibras y la matriz, la crisis máxima puede ser referida a las propiedades mecánicas de las fibras, y eso ocurre mediante la ruptura de las fibras o la delaminación del substrato, como sucede para los materiales epoxi en función de la carga de ruptura de las fibras.

La combinación de los ensayos de tracción cíclico y ensayos de resistencia al cizallamiento llevados a cabo en el laboratorio CIRI de la Universidad de Bolonia (8), permiten discriminar entre los diferentes sistemas de reforzamiento y las tecnologías (FRP o FRCM), alcanzando el primer objetivo de esta investigación: "describir una metodología que permita seleccionar materiales compósitos con propiedades bien definidas que puedan ser un buen instrumento para ser usados por los diseñadores en trabajos prácticos".

Además de las conclusiones anteriores, debemos enfatizar que las resinas Betontex IPN no presentan ninguna temperatura de transición vítrea de segundo orden y muestran una alta resistencia térmica más allá de los $160^{\circ} \mathrm{C}$; además, no se queman, no muestran propagación de flamas y garantizan una alta porosidad y permeabilidad al vapor de agua (MVTR), una propiedad muy importante en el refuerzo de mampostería.

\section{AGRADECIMIENTOS}

El profesor Marco Savoia y a todo el equipo técnico del CIRI -"Buildins and Construction Research Center " de la Universidad de Bolonia por su colaboración y su valiosa discusión de los resultados durante el estudio de las propiedades mecánicas y aplicaciones del sistema IPN.

El profesor Raffaele Poluzzi de la Universidad de Bolonia por el diseño de las primeras aplicaciones de nuestro trabajo de investigación para la rehabilitación de importantes construcciones históricas de la Ciudad de Bolonia.

Al profesor Pierpaolo Diotallevi y a todo el equipo técnico del DICAM de la Universidad de Bolonia por las importantes y exhaustivas pruebas de la aplicación de la tecnología IPN a refuerzos de vigas de concreto.

La profesora Cristina Siligardi, la Dra. Elena Fabbri y el Ing. Cesare Signorini del Departamento de Ingeniería "Enzo Ferrari” por la duradera colaboración en la investigación del material IPN y la investigación de su morfología.

La Dra. Erika I. Cedillo-González del equipo de la Universidad de Módena y Regio Emilia por 
su colaboración en el estudio del sistema IPN y la traducción al idioma español de este artículo.

\section{REFERENCIAS BIBLIOGRÁFICAS}

1. R. Poluzzi- Bolonia University: INARCOS (1) 63-67 Enero 2003.

2. G. Bartoli-Florence University: One Day Presentation of CNR DT 200/2004 Florencia Enero 16,2006 "CR- beams tensile testing after reinforcement by CFRP using "Ardfix" anchoring systems".

3. Ceb/Fib n. 14/2001 "Externally bonded FRP reinforcement for RC structures".

4. Guide Lines CNR document DT200/2004.

5. Ardea s.r.l.- Technical data sheet $\mathrm{n}^{\circ} 7$ bis: " Resine

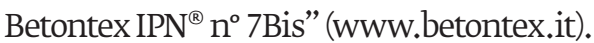

6. L., Credali -EUROPEAN PATENT N ${ }^{\circ}$ EP0994223 B1Priority :October 13, 1998 IT.
7. Ardea s.r.l-Betontex Guide: "Guida ai Prodotti Betontex IPN ${ }^{\circledR}$ ” September 2012 Edition (www. betontex.it).

8. M. Savoia and others-CIRI University of Bolonia : "Laboratory testing on Betontex IPN ${ }^{\circledR}$ laminates", Reports $n^{\circ}$ 142-269-483- Ardea s.r.l. -Research Programs on Mechanical Testing of IPN laminates (2013-2014).

9. P.Diotallevi and others, University of Bolonia : "Application of IPN water-based resins in reinforcement of RC beams by FRP composite materials" - International Conference on Shells Plates and Beams ,Bolonia, It, September 9-11, 2015.

10. L. Credali- IT PATENT: No ITR0001338380- January 22, 2002; Betontex Guide: "Ardfix Connection Elements for Construction" (www.betontex.it). 